Author Accepted Version. Final version published as: Temple, J. B., Kelaher, M., Utomo, A., Williams, R., \& Brooke, L. (2020). Components of disability exclusion: Discrimination, avoidance and accessibility in later life. Australasian journal on ageing, 39(2), 101-111.

\title{
Components of Disability Exclusion: Discrimination, Avoidance and Accessibility in Later Life
}

\begin{abstract}
(150 words)
Objective: Examine the prevalence and context of disability exclusion reported by people with disability, with and without communication and mobility problems.

Methods: The 2015 Survey of Disability Ageing and Carers was used to measure the prevalence of reported exclusion. Tests of proportions were used to examine differences in prevalence rates. Proportional Venn diagrams were used to examine the intersection of measures of exclusion.
\end{abstract}

Results: Approximately 53\% of those with a communication or mobility difficulty reported at least one measure of exclusion. Among this group, 7\% reported an instance of discrimination, $44 \%$ reported avoidance and $29 \%$ reported an accessibility problem. The levels of discrimination and avoidance for those without communication or mobility difficulties was approximately half this level (16\% avoidance and $3 \%$ discrimination). Avoidance and discrimination reduced with age, but accessibility was age-invariant. Exclusion measures intersected and occurred in a variety of contexts.

Conclusion: Despite protections provided in Australian law, disability exclusion persists and is high among those with communication and mobility difficulties.

Keywords: Ageing, Disability, Discrimination, Avoidance, Accessibility. 


\section{Impact Statement (50 words)}

Addressing the multiple and intersecting components of exclusion is crucial to promoting the social and economic inclusion of older Australians with a disability. Current levels of exposure to social exclusion persist despite current protections in Federal and State law, as well as agreements with international accords. 


\section{Components of Disability Exclusion: \\ Discrimination, Avoidance and Accessibility in Later Life}

\section{Introduction}

Approximately $18 \%$ of Australia's population live with a disability, with prevalence rates considerable higher for older Australians [1]. About one quarter of persons aged 55-59 have a disability, rising to $85 \%$ of those aged 90 and over [1]. As Australia's population continues to age, the number of older Australians living with a disability will increase considerably. Giles et al. estimate that over the period $2006-2031$, the number of older Australians living with a profound disability will increase by about $70 \%$ [2]. As these authors note, this projected demographic change poses important implications for families, friends, volunteers and paid service providers with respect to increased demand for assistance and care.

Another important implication of this demographic change is the need to promote social and economic inclusion of this growing population. Many member OECD (Organisation for Economic Cooperation and Development) countries are altering their policies for people with disabilities from one of passive support to one that seeks to promote economic and social inclusion [3]. Moreover, many countries including Australia, are signatories to the Convention on the Rights of Persons with Disabilities (CRPD) which seeks to improve human rights outcomes for this population. Notwithstanding this policy shift and agreement with international accords, the experiences of poor treatment that some people with disabilities face has recently become centre stage in Australia culminating in the Government's announcement of a 
Royal Commission into aspects of abuse, violence and neglect in this population in April 2019 [4]. In the context of promoting social inclusion of older Australians with disability, such policy inquiry is a welcomed development following the Royal Commission into Aged Care Quality and Safety that began in October 2018 [5].

Experiencing discrimination is antithetical to the goals of promoting social and economic inclusion of people with disabilities. In this paper, we utilise 'disability exclusion' as an umbrella term covering exposure to discrimination, avoidance or accessibility problems due specifically to disability. Recent data collections by the Australian Bureau of Statistics (ABS) which have operationalised each as follow:

- Discrimination: Occurs when people reported "felt they had been unfairly considered or treated due to their disability" [1].

- Avoidance: Occurs when people "not going or staying away from people or places because of one's disability" [1]

- Accessibility: Occurs when people "had difficulty accessing locations because of [their] conditions" [6].

Several recent studies have examined aspects of disability discrimination and avoidance in Australia. In a study of adults, Temple et al. (2018) found that about 8.6\% of persons with a disability aged 15 and over in experienced discrimination and almost one-third avoided situations because of their disability [7]. Both discrimination and avoidance has been found to vary over the life course, with younger Australians with a disability more likely to report either discrimination or avoidance relative to older Australians [7]. In another study, Temple and Kelaher (2018) found a strong association between experiencing disability discrimination or avoidance and psychological distress [8]. A 
study focusing on older Australians specifically, found that experiencing disability discrimination or avoidance doubled the probability of experiencing psychological distress and that the effects were heightened for those with specific disabilities such as sensory, speech and physical disabilities [9]. These Australian studies on disability reinforce the large evidence base showing discrimination (in its many forms) is an important social determinant of health and is associated with a wide range of deleterious health outcomes $[10,11]$.

Despite the contribution of these population level studies, there remains a gap in our understanding about the experiences of disability exclusion reported by older persons themselves, particularly for those facing mobility and communication difficulties - a group for whom the effects of discrimination may be more pronounced [9]. Moreover, there remains a lack of knowledge about the multiple forms of exclusion - including discrimination, avoidance and accessibility problems - faced by this population. In this paper, we seek to answer four questions about older Australians reports of exclusion attributable to their health conditions. First, how prevalent are reports of discrimination, avoidance and accessibility? Second, are specific demographic groups more likely to self-report experiences of exclusion across these domains? Third, in which contexts or situations does exclusion occur? Finally, do these multiple forms of exclusion intersect (i.e., across all domains of discrimination, avoidance and accessibility)?

\section{Methods}

\section{Data}

Data for this study are from the 2015 Survey of Disability, Ageing and Carers (SDAC) conducted by the Australian Bureau of Statistics (ABS) between July and December 
2015. SDAC collected information from persons living in private dwellings, in selfcare retirement villages and in cared accommodation. The modules measuring disability exclusion were collected in the household component of the survey - which included individuals living in both private dwellings and self-care retirement villages. Of 31,957 total households originally contacted for SDAC, 25,555 fully responded, yielding a response rate of $80 \%$.

The ABS has the authority to collect these data under the Census and Statistics Act 1905. Data for this study were made available to the authors through the ABS and Universities Australia agreement and ethics approval for this project was granted by the Melbourne School of Population and Global Health Human Ethics Advisory Group (HEAG) - Ethics ID: 1953686.1.

\section{Measurement of Exclusion}

Operationalisation of disability exclusion is available in SDAC through the concepts of discrimination, avoidance and accessibility. All three concepts relate to respondents self-reported exposure. The measure of discrimination is based on response to the question "In the last 12 months do you feel that you have experienced discrimination or have been treated unfairly by others because of your condition/s?" For those who responded 'yes', a follow up question was asked: "Who treated you unfairly or discriminated against you because of your condition/s?” A list of multiple responses was provided (available in Table 2).

The measurement of avoidance is based on response to the question "In the last 12 months have you avoided situations because of your condition(s)?" Again, those who 
responded 'yes' were asked the following question: "What situation(s) did you avoid because of your condition(s)?" A list of multiple responses was provided (available in Table 2).

As detailed below, the ABS identified a group of persons living with a disability at a heightened risk of accessibility problems. These included those with assistance needs or difficulties associated with mobility or communication problems and were asked to respond to an additional accessibility module (in addition to the avoidance and discrimination questions). This population was asked to respond to the following question: "In the last 12 months, have you had difficulty accessing locations because of your disability"? Two follow up questions were asked regarding the types of difficulties (e.g., inadequate lifts of escalators, bathroom facilities), and the location in which the person experienced the accessibility difficulty (available in Table 2).

\section{Population}

In the following analyses, two populations are examined in detail:

- Sample 1 - A sub-sample of $n=2091$ responding to the accessibility module in SDAC who (1.) need assistance or have difficulty with communication with someone they do not know because of disability, or (2.) need assistance or have difficulty with mobility because of disability. This group represents $32 \%$ of the sample of aged 55 and over living with a disability in households. This group answered questions relating to discrimination, avoidance and accessibility.

- Sample 2 - A sub-sample of $n=4442$ persons living with a disability in private households aged 55 and over, but not included in sample 1. This group answered questions relating to avoidance and discrimination only. 
- Total Sample - The total sample consisted of $n=6533$ (samples 1 and 2) persons living with a disability in private households aged 55 and over.

Within and between these populations, Wald tests of proportions were conducted to measure differences in prevalence rates of exclusion. The prevalence rates were weighted to adjust for non-response. Proportional Venn diagrams are presented to depict the intersection between discrimination, avoidance and accessibility.

\section{Results}

Prevalence of Exclusion

Approximately $4.5 \%$ of older Australians living with a disability in households reported an instance of discrimination in the last 12 months and $25 \%$ reported avoiding a situation or context due to their disability (Table 1). When stratified by sample, a clear difference in propensities of exclusion are observed. Of those with communication and mobility difficulties, approximately $7 \%$ reported an instance of discrimination, $44 \%$ reported avoidance and 29\% reported an accessibility issue in the last 12 months (sample 1). The levels of discrimination and avoidance for those without communication or mobility difficulties are approximately half this level $(16 \%$ avoidance and $3 \%$ discrimination - sample 2).

\section{Demographic Differences in Exclusion}

Although these differences between those with and without communication and mobility difficulties are significant, we also observe significant differences in the prevalence of exclusion by demographic and health characteristics within each sample. For discrimination, we identify a number of sub-populations with a prevalence rate 
more than twice the population levels rate (i.e., $9 \%$ or more). For both samples, these include the unemployed, and those living with intellectual or psychosocial disabilities. For those with communication and mobility difficulties (sample 1), high discrimination prevalence is observed for those in the youngest age group $(55-64=13.6 \%)$, persons who are separated, divorced or never married $(12-16 \%)$, those with a head injury $(15.2 \%)$, other disability $(9.3 \%)$ or with 3 or more disability types $(11.5 \%)$.

The heightened risk of reported exclusion of these groups is also reflected in the prevalence of avoidance, but at a substantially higher level. In particular among those with communication and mobility difficulties, avoidance is cited by more than half of those in the aforementioned demographic and disability type groups. Almost two thirds of those with communication or mobility difficulties living with a psychosocial disability reported avoidance $(64 \%)$.

Restricting the analysis to those with communication and mobility difficulties (sample 1), there are some notable differences and similarities with respect to demographic groups at risk of accessibility problems. Whereas both discrimination and avoidance reduced with age for both samples, accessibility problems are invariant by age (approximately 30\%). Whereas region of residence did not differentiate avoidance or discrimination, a slightly higher proportion of persons in cities (31\%) reported accessibility difficulties relative to those in inner regional $(26 \%)$ or other $(25 \%)$ areas of Australia.

Females relative to males were also more likely to cite accessibility ( $32 \%$ and $24.8 \%$ respectively). Again, persons with psychosocial and physical disabilities are 
highlighted as a group at increased risk of accessibility problems, as are those with multiple disability types.

\section{Contexts and Situations of Exclusion}

These demographic and health differences in the prevalence of exclusion raise the question of where it occurs? Table 3 displays the source of discrimination, situation of avoidance and facility in which respondents had accessibility problems.

For those without communication or mobility difficulties, the highest ranked sources of discrimination include 'other' (30\%), strangers in the street (18\%) and family and friends (19\%). Just under 1 in 5 cite employer (16.2\%). For those with communication or mobility problems, reports of discrimination in everyday social contexts is also observed. However, almost one in four report health staff as a source of discrimination (22.1\%), compared to about $12 \%$ of those in sample 2.

Situations avoided also mirror everyday social contexts as observed with discrimination. For example, around $45 \%$ of both samples cite 'other social situations'. However, those with communication and mobility difficulties are more likely to avoid a range of everyday social situations. Over $30 \%$ report avoidance of visiting family or friends (39\%), shops and banks (37\%), restaurants (33\%), public transport (36\%) and other public places (32\%). Interestingly, although cited discrimination in healthcare contexts was relatively high for this group compared to those without communication and avoidance difficulties, avoidance is lower (around $6-7 \%$ ). Around $11 \%$ of the full sample cite avoidance of the workplace. 
Turning to facilities in which people had accessibility concerns, the majority reported commercial settings such as shops and banks (62\%). Around 20 to 30 percent of respondents cite other facilities related to socialisation including other people's homes $(27 \%)$, restaurants or cafes $(28 \%)$, public parks or recreation venues $(18 \%)$ or cultural pursuits $(25 \%)$. Concerningly, almost $40 \%$ of those in sample two note medical facilities (37.3\%) and approximately one quarter report Government buildings (25\%).

\section{Multiple Instances of Exclusion}

Our final research question was to investigate how reports of multiple exclusion interact. The experiences of discrimination and avoidance (Figure 1 - full sample) and discrimination, avoidance and accessibility (Figure 1 - sample 1) can conveniently by summarised using proportional Venn diagrams.

Firstly, for those with a communication or mobility difficulty, the thick lined rectangle represents the total population (Figure 1 - sample 1). The dashed circle represents avoidance (44.3\%), dotted circle discrimination (7.4\%) and solid circle accessibility difficulties $(31.5 \%)$. In total, approximately $53 \%$ of those with a communication or mobility difficulty reported at least one measure of exclusion. It is clear that a considerable degree of overlap exists between the three groups. Of those citing avoidance, $14 \%$ report discrimination and $45 \%$ report accessibility difficulties. Of those citing accessibility difficulties, 13\% report discrimination and 68\% cite avoidance. Finally, of those citing discrimination, 51\% report accessibility difficulties and $81 \%$ report avoidance. Approximately half of those reporting avoidance report this form of exclusion only $(49.6 \%)$ - with no discrimination or accessibility problems. The 
proportions are lower for discrimination (14.9\%) and access difficulties (12.0\%). That is the later two groups are more likely to report multiple levels of exclusion.

For the full sample (Figure 1 - full sample) and sample of those without a communication or mobility difficulty (Figure 1 - sample 2), a considerable overlap between the concepts of avoidance and discrimination also exist. Of those reporting discriminations, 74\% (full sample) and 67\% (sample 2) concurrently reported avoidance.

\section{Discussion}

Results from this study underscore the substantial levels of exclusion faced by older Australians living with a disability, with the situation particularly marked for those with communication and mobility difficulties. Almost half of this population reported some form of exclusion (either discrimination, avoidance or accessibility problems). This is consistent with evidence underscoring barriers to living independently and participation in community life facing persons with communication and mobility difficulties [12].

Our findings here are concerning given that Australia has a legal framework consisting of anti-discrimination laws at the Commonwealth, State and Territory levels to protect the rights of people living with disabilities in addition to being a signatory to the CRPD. Clearly, exposure to discrimination, experiencing accessibility problems and engaging in avoidance behaviours because of disability is antithetical to the aims of this legal framework. 
Our analyses of sources of reported discrimination, situations avoided and facilities with accessibility problems underscore two facts. First, much of the level of exclusion is in everyday social and familial situations. Second, exclusion takes place in contexts critical to human capital - health care, government buildings and to a lesser extent in this older population, the workplace. This is a finding that has been observed for other forms of discrimination in later life [13]. A peculiar observation was also found for exclusion and health care; for those with communication and mobility difficulties, heightened levels of discrimination and accessibility problems were cited, but not for avoidance. We hypothesize that this is likely due to the fact that this group has no choice but to attend to their health conditions - regardless of problems they may face in doing so. For some, their health conditions may push them into contexts where they know they may be exposed to discrimination or encounter significant accessibility problems.

We further demonstrate that reported exclusion is higher in certain groups, including those who are not married (relative to the married), the unemployed (relative to the employed) and those living with multiple disabilities and specific disabilities such as psychosocial and intellectual disabilities. These levels of heightened risk have been observed in the broader Australian population and highlight the association between indicators of socio economic and health status and exclusion [7]. Interestingly, although both discrimination and avoidance falls with age in both samples, accessibility remains invariant by age.

Research on other types of discrimination suggests that exposure varies with age. Exposure to racism [13-15] and sexism [14] decreases throughout adulthood while ageism not surprisingly increases with age [14]. Avoidance of discrimination would 
accordingly also be expected to decrease with age. There are two key explanations for these differences. First, it may be that there are actual changes in exposure to discrimination [14]. Second, exposure to discrimination may be normalised throughout the life-course rendering its occurrence unremarkable and attenuating its impacts [14]. This may be even more likely to be true in the case of disablism because disability becomes far more common and hence normalised with age. In contrast accessibility is a feature of environments rather than individuals. It would not be expected that this would vary as people age.

Finally, we sought to understand the prevalence of multiple forms of exclusion reported by older people living with as disability. The concepts of discrimination, avoidance and accessibility we employ are consistent with the social model of ageing and disability. At the heart of the social model is promoting the social inclusion of older people living with a disability. As argued by Sargeant (2011) [17, p 9-10]:
"The closeness of age and disability as issues is perhaps further illustrated when considering the medical and social models of disability. The medical model focuses on the individual and postulates that an individual's inability to work or participate in society is a result of the disability .... It is for the individual to adapt to society's norms. The social model, on the other hand, treats disability as a loss of opportunity to participate in society and work. The problem is not the individual but society. Barriers exist in society which stop people with a disability from fully participating. These barriers can relate to the inaccessibility of the physical environment such as buildings or to the stereotypical attitudes of society about disabled people."

In this paper we have disentangled different aspects of disableism into discrimination and accessibility. Both measures were included because much discrimination against disabled people is enacted by limiting accessibility. Limiting accessibility is generally considered a type of discrimination as suggested in the above quote. The results show that if accessibility is not included separately then the level of discrimination 
experienced by disabled people will be significantly under estimated. Our inclusion of accessibility is important as it is a central component of the World Health Organization's Active Ageing process of optimizing opportunities for health, participation and security in order to enhance quality of life as people age [18]. It is an important precondition for workforce participation of older people with disabilities, to prevent precarity and unemployment $[19,20]$.

Avoidance is a response to discrimination.. Accordingly, failure to take avoidance into account will also lead to discrimination and its impacts being underestimated. The high prevalence of avoidance among people with disability may reflect the extent to which discrimination takes the form of rendering environments inaccessible. Discrimination, accessibility and avoidance are distinct but overlapping concepts which all add to our understanding of the ways in which people with disabilities are excluded by society.

\section{Limitations and Extensions}

Interpretation of our findings needs to be balanced by the limitations of the data and analysis. Firstly, the measures of exclusion are all self-reported and may be subject to bias due to (1.) recall over a 12 month period, (2.) some respondents may feel uncomfortable disclosing instances of exclusion, (3.) not all measures of perceived discrimination may be actual discrimination. A further limitation is that the modules on discrimination and avoidance were only asked of people with disabilities living in households and who responded to a personal interview, potentially biasing prevalence downwards [21]. 
This study has presented a descriptive analysis of aspects of social exclusion reported by older Australians living with a disability. Multivariable studies would provide fruitful insights into the modelling of exclusion jointly as a construct of discrimination, avoidance and accessibility. Future data collections in SDAC could generalise the accessibility module to all persons living with a disability, not just those with communication and mobility difficulties. An important area of future research is the exclusion faced by Aboriginal and Torres Strait Islander people living with disability. Unfortunately, the SDAC CURF (Confidentialised Unit Record File) made available to researchers by the ABS does not include a flag for Aboriginal and/or Torres Strait Islander status. Recent studies highlight that Aboriginal people experience a significantly higher prevalence of disability in conjunction with a unique form of intersectional discrimination' as a consequence [22]. Moreover, our results suggest further research on the economic as well as health consequences of exposure to exclusion by people with disabilities is a pressing issue for further research. Finally, as SDAC was collected against Australian-specific policy and societal contexts, the generalisability of our findings to other countries requires further research.

\section{Conclusion}

Using nationally representative data on older people with disabilities, we demonstrate that (1.) levels of reported disability exclusion (as measured by reported discrimination, avoidance and accessibility) is experienced by a substantial proportion of older Australians with disabilities (2.) that these measures are particularly pronounced for persons with communication and mobility difficulties with 53\% of this group reporting at least one measure of exclusion. and (3.). Reported exclusion occurs in a variety of contexts critical to social inclusion, but also areas critical to human capital including 
health care, government building and for some, the workplace. Current levels of exposure persist despite current protections in Federal and State law, as well as agreements with international accords.

\section{References}

1. ABS. Disability, Ageing and Carers, Australia: Summary of Findings, 2015. Catalogue Number 4430.0, Australian Bureau of Statistics: Canberra, 2016. [Cited 1 February 2019]. Available from URL: https://www.abs.gov.au/ausstats/abs@.nsf/mf/4430.0

2. Giles L, Cameron I, Crotty M. Disability in Older Australians: projections for 2006 to 2031. Medical Journal of Australia 2003; 179: 130-133.

3. OECD. Modernising sickness and disability policy: OECD thematic review on sickness, disability and work. Issues paper and progress report. OECD: Paris, 2008. Available from URL http://www.oecd.org/employment/emp/40495992.pdf

4. Commonwealth. Royal Commission into Violence, Abuse, Neglect and Exploitation of People with Disability [Cited 12 August 2019]. Available from URL: https://disability.royalcommission.gov.au/Pages/default.aspx Date accessed August 2019.

5. Commonwealth. Royal Commission into Aged Care Quality and Safety [Cited 12 August 2019]. Available from URL https://agedcare.royalcommission.gov.au/Pages/default.aspx. Date accessed August 2019.

6. ABS. Survey of Disability, Ageing and Carers: Household Survey Questionnaire. Australian Bureau of Statistics, Canberra, 2015 [Cited 1 
February 2019]. Available from URL:

http://www.ausstats.abs.gov.au/ausstats/subscriber.nsf/0/8F411A618C3CD63 DCA25804F000F60CD/\$File/attk7lrm.pdf. Date accessed February 2019.

7. Temple J, Kelaher M, Williams R. Discrimination and avoidance due to disability in Australia: Evidence from a national cross sectional survey. BMC Public Health 2018; 18:1347.

8. Temple J, Kelaher M. Is disability exclusion associated with psychological distress? Australian evidence from a national cross-sectional survey. BMJ Open 2018; 8:e020829.

9. Temple J, Kelaher M, Williams R. Disability discrimination and avoidance in later life: Prevalence, disability differentials and association with mental health. International Psychogeriatrics 2019; In Press

10. Pascoe E, Smart Richman L. Perceived discrimination and health: A metaanalytic review. Psychological Bulletin 2009; 135: 531-554.

11. Paradies Y, Jehonathan B, Denson N, Elias A, Priest N, Pieterse A, Gupta A, Kelaher M, Gee G. Racism as a determinant of health: A systematic review and meta-analysis. PLOS One 2015; 10(9): e0138511

12. Australian Government. Shut Out: The experience of people with disabilities and their families in Australia, National Disability Strategy Consultation Report prepared by the National People with Disabilities and Carer Council, 2009.[Cited 25 March 2019]. Available from URL https://www.dss.gov.au/sites/default/files/documents/05_2012/nds_report.pdf,

13. Temple J, Kelaher M, Paradies Y. Prevalence and context of racism experienced by older Aboriginal and Torres Strait Islanders. Australasian Journal on Ageing 2019; 38(1):39-46. 
14. Gee GC, Hing A, Mohammed S, Tabor DC, Williams DR. Racism and the Life Course: Taking Time Seriously. American Journal of Public Health. 2019;109(S1): S43-S7.

15. Greer TM, Spalding A. The role of age in understanding the psychological effects of racism for African Americans. Cultural Diversity \& Ethnic Minority Psychology 2017;23(4):588-94.

16. Markwick A, Ansari Z, Clinch D, McNeil J. Experiences of racism among Aboriginal and Torres Strait Islander adults living in the Australian state of Victoria: a cross-sectional population-based study. BMC Public Health 2019;19(1):309-.

17. Sargeant M. 'Ageism and age discrimination' in Sargeant M (ed). Age Discrimination and Diversity: Multiple Discrimination from an Age Perspective. Cambridge University Press: Cambridge, 2011

18. World Health Organization. Active Ageing: A Policy Framework. World Health Organization, 2002.

19. Shuey, KM, Jovic, E. Disability Accommodation in Nonstandard and Precarious Employment Arrangements, Work and Occupations 2013; 40 (2): 174-205.

20. Australian Human Rights Commission. Willing to Work, National inquiry into employment discrimination against older Australians and Australians with a disability. Australian Human Rights Commission, 2016.

21. Temple JB, Stiles J, Kelaher M, Utomo AJ, Williams R. Is disability exclusion associated with experiencing unmet needs to healthcare. Australasian Journal on Ageing 2019; under review. 
22. Avery S. Culture is inclusion: A narrative of Aboriginal and Torres Strait Islander people with disability. First Peoples Disability Network (Australia). Sydney, Australia, 2018. 


\section{University Library}

\section{- M M N E R VA A gateway to Melbourne's research publications}

Minerva Access is the Institutional Repository of The University of Melbourne

Author/s:

Temple, JB;Kelaher, M;Utomo, A;Williams, R;Brooke, L

Title:

Components of disability exclusion: Discrimination, avoidance and accessibility in later life

Date:

2019-11-18

Citation:

Temple, J. B., Kelaher, M., Utomo, A., Williams, R. \& Brooke, L. (2019). Components of disability exclusion: Discrimination, avoidance and accessibility in later life. AUSTRALASIAN JOURNAL ON AGEING, 39 (2), pp.101-111. https://doi.org/10.1111/ajag.12750.

Persistent Link:

http://hdl.handle.net/11343/253845 